\title{
Exploration Practice of Diagnosis, Treatment and Prevention of Bovine Brucella
}

\author{
Yang Meng, He Zhuqing \\ School of Bioengineering, Xi' an Vocational and Technical College, Xi'an, 710077, China
}

\begin{abstract}
Bovine brucellosis is bovine brucellosis, a zoonotic infectious disease. It has the characteristics of wide distribution and great harm, which seriously hinders the improvement of economic benefits of the cattle industry. With the rapid development of economic exchanges, livestock breeding and trading activities between regions have become more frequent, and the epidemic of bovine brucellosis has gradually spread. China's "Animal Epidemic Prevention Law" stipulates brucellosis as a second-class infectious disease, which is caused by Brucella. Cattle infections can cause abortion, stillbirth, infertility, lameness and orchitis, which pose a huge threat to the development of the breeding industry. With the continuous expansion of the number and scale of domestic cattle raising, the epidemic situation of brucellosis has rebounded. This article is mainly aimed at the diagnosis, treatment and prevention of bovine brucellosis, and puts forward opinions and suggestions based on the actual situation.
\end{abstract}

\section{Introduction}

After 40 years of research, China's research on brucellosis has made great achievements. The effect of disease prevention and control has improved significantly, but it is far from being completely eliminated. Large-scale bovine brucellosis still occurs in many breeding areas, affecting the economic benefits of farmers. Brucellosis is more susceptible to animals, including livestock such as pigs, cattle, and sheep. Brucellosis mainly affects the reproductive system and causes symptoms such as inflammation of the membranes, infertility, and orchitis. Humans will be infected with brucellosis by contacting items carrying pathogenic bacteria, causing infections of the digestive tract and respiratory tract. After infection in humans, symptoms such as chronic low fever, general fatigue, joint pain, and orchitis may occur. Brucellosis poses a huge threat to livestock production and human health and requires multiple measures to actively control it.

\section{Pathogens and Epidemic Characteristics}

Brucella is a small coccus that appears rod-shaped after subculture. The germs have no flagella, no spores, sterile hairs, do not move, and no capsules will appear in most logarithmic cases. There are six species of Brucella. Brucella abortus, Brucella malta, and Brucella suis are responsible for disease in cattle. Brucellosis occurs frequently in farming areas and is mainly concentrated in the litter stage. Cows are more susceptible to infection and become more pronounced after sexual maturity. Diseased animals and carrier animals are the main source of infection. After cow abortion, the bacteria will be discharged with the placenta and fetus, and then become the strongest source of infection in the region. The digestive tract is the main route of Brucella infection, and infection can also occur through damaged skin, mucous membranes, and mating. Brucella is very invasive and can even invade directly from healthy skin and cause infections.

Diseased and carrier cattle are the main source of infection of brucellosis, especially the fetus and various secretions after cow abortion contain a large number of pathogenic bacteria. In addition, a large number of pathogenic bacteria will appear in the bull's semen. Substances that carry pathogenic bacteria will cause pollution to the environment in the breeding area and will easily cause large-scale epidemics. Bovine brucellosis is mainly transmitted through digestion, and cattle develop infection symptoms after eating contaminated feed. It may also be infected when it comes into contact with aquaculture-carrying objects, or if the breeding process or artificial disinfection is not strict. The management conditions in the breeding area are not up to standard. Cattle and blood are not handled properly in the slaughter stage, and cattle with weak resistance are very susceptible to infection. The probability of infection increases with the maturity of the bovine sex organs, and the calves have corresponding resistance. 


\section{Clinical Symptoms and Pathological Changes}

Bovine brucellosis has an incubation period of 2-24 weeks, and most cases are negative infections. Pregnant cows are susceptible to miscarriage, and abortions can occur at any time. It usually occurs from June to August after pregnancy, and the survival rate of aborted fetuses is very low. Cows infected with brucellosis may experience childbirth before abortion and swelling of the labia and breasts. There will be red nodules the size of millet grains in the vaginal mucosa, and gray-white viscous secretions will flow out of the vagina. Cows may have symptoms such as retention of placenta and endometritis after abortion. In the next 1-2 weeks, red-brown secretions will be discharged from the vulva and have a foul smell. Cows will develop mild mastitis after infection, and joints and synovial bursitis may occur in some cases. Bull infections can cause orchitis and epididymitis. Sometimes the symptoms of nearby lymph nodes are enlarged, and the penis is flushed. The bull's sperm activity is reduced, and the mating success rate is significantly reduced.

A necropsy revealed a noticeable thickening of the placenta, which was accompanied by bleeding points. There is a yellow gel on the surface, along with fibrin and pus. After the abortion of the cow's uterus, there will be gray exudates between the mucous membranes and chorion, and the aborted fetus will develop sepsis. The aborted fetus produces floes in the stomach and is accompanied by white mucus. A large amount of red effusion appeared in the thoracic cavity, and there was serous infiltration of subcutaneous and connective tissue. There are bleeding spots in the serosa and mucous membranes, and bleeding spots may be found under the skin and between the muscles. The lymph nodes, liver, and spleen of diseased cows will gradually swell, and granulomas will gradually form. Cows have substantial mastitis, followed by secondary atrophy and sclerosis of the mammary glands. Bulls can have necrotizing orchitis and epididymitis, and sometimes necrotic foci.

\section{Diagnostic Characteristics of Bovine Brucellosis}

\subsection{Etiological Examination}

Smears were made using abortion fetal garments, cow vaginal secretions, and milk, and examined by Coriolis staining. First fix the smear, add $0.5 \%$ sand yellow solution dropwise, and heat up for 2 minutes to get air bubbles. Then, $0.5 \%$ malachite green solution was added dropwise by washing, and dyeing was performed for $45 \mathrm{~s}$. Finally, it was washed, dried, and examined by microscopy. After the test, the brucella appears red, and the other bacteria appear green. Brucella clusters in cells, and only a few are outside the cell.

\subsection{Separation Culture}

Brucellosis requires oxygen for reproduction, and it also has strict nutritional requirements. When performing isolation culture, select a serum-rich medium. Add 3\% bovine serum to the basal medium, or you can use serum glucose agar instead. Brucella grows slowly and can only be observed with the naked eye after one week of culture.

\subsection{Differential Diagnosis}

Clinically, abortion in cows can be divided into infectious and non-infectious factors. Infection of Campylobacter and Corynebacterium pyogenes may also cause abortion in cows, which need to be effectively distinguished in clinical symptoms. At this time, the smears of the aborted fetus and the cow's guided secretions should be used to make a smear based on laboratory etiology diagnostic techniques to observe the morphology of the bacteria.

\section{Control of Bovine Brucellosis}

\subsection{Establishing A Comprehensive Disease Prevention and Control Mechanism}

Brucella is a facultative intracellular parasite, and the effect of drug treatment is not obvious. Therefore, prevention-oriented, timely vaccination to avoid the spread of immune status. The prevention of any disease requires capital and human investment, and bovine disease is no exception. Relevant departments have special financial support for the prevention and control of brucellosis, but the actual funding gap is large. It is generally used to purchase detection reagents, and compensation for culling sick animals is very small. There are many obstacles to the establishment of prevention and control systems, and the effectiveness of epidemic prevention and control is very limited. In order to further improve the level of prevention and control of the epidemic, it is necessary to build a comprehensive mechanism for the prevention and control of brucellosis to reduce the harm of brucellosis.

\subsection{Regular Quarantine}

In areas not endemic for brucellosis, quarantine should be guaranteed once a year. When introducing new cattle, two serum tests should be used, and the animals should be kept in isolation for two months before being put into the herd. For the frequently-occurring areas of the disease, quarantine should be carried out twice a year, respectively in spring and autumn. Cows who have been vaccinated should be quarantined here within 3 years after vaccination. Once positive cattle are found, they need to be processed in time. If the disease is mild, isolation breeding treatment should be adopted, and at the same time, the treating staff should do their own protection. For diseased cows with lower therapeutic value, pollution-free treatment measures must be adopted. 


\subsection{Strengthen the Professional Team \\ Construction of Bovine Brucellosis \\ Prevention}

The economic loss caused by bovine brucellosis has caused many negative impacts on the development of the breeding industry. There are many factors that have repeatedly banned the epidemic. Inadequate professional knowledge of farmers and poor awareness of epidemic prevention are important reasons. In addition, grassroots animal epidemic prevention professionals are inadequate, and there are many restrictions on epidemic prevention of bovine brucellosis. It is necessary to analyze the current situation of the personnel in the epidemic prevention station, and to cultivate highly professional and highly epidemic prevention personnel. Regularly quarantine the epidemic situation of brucellosis of local animals and personnel, and timely predict the epidemic trend of brucellosis.

\section{Conclusion:}

In summary, it is very important to explore the practice of diagnosis, treatment and prevention of bovine "bout disease". Emphasize the prevention of epidemic diseases, and formulate detailed epidemic prevention regulations and work requirements. Further promote publicity and education on epidemic prevention knowledge and guide farmers to actively cooperate with animal epidemic prevention work.

\section{References:}

1. Cheng Wenjie. Diagnosis, treatment and prevention of bovine brucellosis [J] Shandong Animal Husbandry and Veterinary Medicine, 2017 (4): 114

2. Shi Xintao, Gu Shaopeng, Zheng Mingxue, et al. Survey of epidemic and control of brucellosis [J] China Animal Husbandry and Veterinary Medicine, 2018 (3) 204-207

3. Zhang Weimin, Zhang Changqian. Diagnosis and prevention measures of bovine brucellosis [J] Modern Agriculture, 2017, 32 (2): 38-39

4. Li Hongke, Li Tiewang. Research on the prevention and control of cattle and sheep brucellosis [J] China Journal of Cattle Industry. 2017 (37) 68-69 